\title{
Healthy behaviours and COPD
}

\author{
Isabella Annesi-Maesano $0^{1,2}$ and Nicolas Roche $e^{3,4}$
}

Affiliations: ${ }^{1}$ EPAR, UMR-S 1136 IPLESP INSERM, Paris, France. ${ }^{2}$ EPAR, UMR-S 1136 IPLESP UPMC Sorbonne Universités, Paris, France. ${ }^{3}$ Service de Pneumologie et Soins Intensifs Respiratoires, Groupe Hospitalier Cochin Broca Hôtel-Dieu, AP-HP, Paris, France. ${ }^{4}$ Université Paris Descartes (EA2511), Paris, France.

Correspondence: Nicolas Roche, Pneumologie et Soins Intensifs Respiratoires, Groupe Hospitalier Cochin Broca Hôtel-Dieu, Site HIA du Val de Grâce 4eC, 74 Bd de Port Royal, 75005 Paris, France.

E-mail: nicolas.rochedacch.aphp.fr

○ @ERSpublications

Healthy behaviours (diet and activity) could have beneficial effects on the risk of COPD occurence or progression http://ow.ly/AhjNh

In this issue of the European Respiratory Review, Young and Hopkins [1] elegantly discuss how beans (an important part of the Hispanic diet) could reduce systemic inflammation and, thereby, the risk of chronic obstructive pulmonary disease (COPD) and lung cancer, explaining the risk imbalance between Hispanics, African-Americans and non-Hispanic whites. As appropriately acknowledged by the authors, what they propose is a hypothesis, warranting confirmation in specific observational and interventional studies. The purpose of this editorial is to discuss some important issues evoked in their reasoning, focusing on COPD only. Altogether, the main question is: is causal inference between bean intake and reduction in the risk of COPD occurrence possible from available data and, if not, what would be needed to progress, considering possible confounders?

\section{Assessing the causality of the link: back to basics}

In 1965, Sir Bradford Hill, an epidemiologist, proposed to consider the following criteria when assessing the causality of a relationship between two events/conditions [2]: 1) the strength of the association, as assessed by statistics (rate ratios, risk ratios and odds ratios);2) consistency and repeatability of the association; 3) specificity of the relationship (one cause leads to one effect); 4) temporal relationship (the cause precedes the consequence); 5) presence of a dose-response relationship, i.e. greater exposure is associated with greater incidence of the effect; 6) biological plausibility; 7) coherence between epidemiological and biological/laboratory evidence; 8) experimental evidence; and 9) analogy with the effect of other, similar factors. Two other factors need to be considered: 1) the possible involvement of bias by confounding factors in the observed association(s) [3]; and 2) the specific issue of confirmation of observations by interventional approaches [4]. The need to consider the criteria of HILL [2] as a guide rather than as a definitive tool has been underlined previously, especially in the area of nutritional epidemiology [5]. Bearing these considerations in mind, what is the evidence linking diet and COPD occurrence, and how can other dietary components or non-dietary factors confound the possible link between bean consumption and COPD?

\section{Biological plausibility}

In COPD, the main dietary components that have attracted interest are fibre (mostly contained in fruits and vegetables, including beans), polyunsaturated fatty acids (PUFAs) and antioxidant micronutrients (vitamins A, C, D and E, and carotenes and flavonoids), as recently reviewed elsewhere [6,7]. These components are actually inter-related: for instance, fermentation of water-soluble fibres in the large intestine promotes the

Received: June 162014 | Accepted: June 172014

Conflict of interest: None declared.

Provenance: Submitted article, peer reviewed.

Copyright OERS 2014. ERR articles are open access and distributed under the terms of the Creative Commons Attribution Non-Commercial Licence 4.0. 
formation of short-chain fatty acids, while most fibre-containing foods (e.g. fruits and vegetables) also contain antioxidant micronutrients.

Regarding biological plausibility, two aspects need to be considered: 1) the involvement of inflammation and oxidative stress in COPD pathophysiology; and 2) the anti-inflammatory/antioxidant effects of the nutrients of interest. Both have been extensively studied, and there is no doubt as to their reality. Indeed, following available data on COPD pathophysiology, anti-inflammatory and/or antioxidant approaches represent by far the most intensively explored ways of affecting the natural history of the disease [8]. Shortchain fatty acids have anti-inflammatory, antioxidant, immune-modulatory and anti-proliferative effects, as highlighted in the paper by YounG and HopkINS [1] and demonstrated in experimental models and in vivo observations. Epidemiological and pathophysiological inter-relations between all the above-mentioned nutrients obviously make it difficult to determine which one is the most important. While the epidemiological evidence specifically regarding beans is limited, relatively large amounts of data are available for other nutrients, which are summarised below since some might interact with bean consumption.

\section{Possible dietary confounders: summary of epidemiological data on diet and nutrition in the prevention of COPD}

COPD has been negatively related to the intake of fruit, vegetables and fish, and positively related to the intake of cured meat, as assessed through a food frequency questionnaire. Intake of antioxidants and PUFAs has been shown to protect against COPD. Most data were cross-sectional and only a few were obtained from prospective cohort studies or case-control studies, thus findings are sparse and not robust. Few intervention studies have evaluated the impact of food or antioxidants on COPD.

\section{Assessment of food and nutrients intake}

The assessment of usual food and energy intake in humans is a hard task, requiring the accurate determination of the subject's routine intake, the conversion of this information to energy and nutrients, and a sufficient duration of food intake measurement to consider the information as a true reflection of habitual food intake. Several methods have been implemented. The Food Frequency Questionnaire (FFQ) and semi-quantitative FFQs are amongst the most used tools in population studies because they allow the collection of dietary information from large numbers of individuals and are normally self-administered. The FFQ is a list of foods and a selection of options relating to the frequency of their consumption in order to capture usual intake. Semi-quantitative FFQs are questionnaires that also collect information about portion size. Where information on portion size is not obtained, standard food portion sizes are often used to calculate nutrient intakes, as is done for many of the FFQs based on the Caerphilly questionnaire [9]. Some of these questionnaires also assess vitamin and PUFA supplementation. A limitation of diet assessment is the potential measurement error in the estimation of food intake. However, any such misclassification is probably non-differential and therefore would lead to an underestimation of the effects of food.

\section{Fruit and vegetables}

Fruits and vegetables have captured most of the interest, given their antioxidant properties and thus their potential anti-inflammatory effect. Several cross-sectional studies showed a significant positive association between the quantity of fruit intake and the risk of COPD or forced expiratory volume in $1 \mathrm{~s}$ (FEV1) reduction [10]. In two studies, the consumption of apples and pears, and the intake of flavonoids were positively associated with FEV1 [11, 12]. An association between a reduced risk of COPD and a high consumption of vegetables has been found in only a few studies [13, 14]. In prospective cohorts, a significant reduction in the risk of COPD was observed with regular consumption of fruit but not vegetables [15-18]. Finally, a case-control study [19] found a significantly lower intake of vegetables and fruit in COPD patients than in controls. In another case-control study in smokers, a high consumption of vegetables and fruit (in particular apples) was associated with a COPD risk reduction [20]. Overall, the strength of the evidence linking a high intake of vegetables and fruit to a reduced risk of COPD is considered as moderate (i.e. there is a possible link), but it needs to be confirmed by other longitudinal data.

\section{Meat}

A traditional diet including a high intake of meat and potatoes and a lower intake of soy and cereals has been associated with a lower FEV1 and a higher prevalence of COPD at the population level [21]. In addition, a deleterious effect of meat has been suggested by the association between a high intake of cured meat and the risk of: 1) COPD occurrence [22]; and 2) hospital readmission in COPD patients [23]. This effect has been attributed to nitrites, which are added as preservatives, and colour fixatives during cured 
meat production. These agents could increase the nitrosative stress in the lung via the formation of reactive nitrogen species, causing damage and remodelling of the lung parenchyma.

\section{Fibre}

A positive association was found between a diet rich in refined grains, cured and red meats, desserts, and French fries, and the risk of COPD [24]. This has been confirmed by one of the studies that investigated vegetable intake in detail, and suggested that a diet specifically rich in cereal fibres may reduce the risk of developing COPD [17]. Among specific fibre sources (i.e. cereal, fruit and vegetables), only refined cereal fibre was significantly associated with newly diagnosed COPD, independently of other sources of fibre. Only one study considered beans in detail. HirAyAma et al. [25] found that soy consumption was positively correlated with observed lung function measures even after adjusting for the effects of confounding factors. The mean soy intake was significantly higher among controls than cases. A significant reduction in COPD risk was evident for highest versus lowest quartile of daily intake of total soy bean products. Soy beans contain soy bean trypsin inhibitor, which inhibits enzymatic chymase activity that may intervene in the pathogenesis of COPD.

\section{Vitamins and PUFAs}

Among antioxidant vitamins, relationships with lung function have been found for vitamin $\mathrm{C}$, vitamin $\mathrm{E}$ and $\beta$-carotene intake. All are protective, although some studies challenged the association between vitamin E consumption and higher lung function [26].

The relationship between PUFAs and COPD prevalence or severity at the population level has not been investigated. Fatty acids have been taken as a proxy for fish consumption. A high intake of $\omega-3$ fatty acids did not appear to protect against COPD, but a high intake of several $\omega-6$ fatty acids was associated with a significant reduction in FEV1, particularly in smokers [21]. Interestingly, it has been shown that PUFA supplementation exerts beneficial effects on exercise capacity in COPD patients undergoing a rehabilitation programme [27]. This observation leads us to evoke the possible role of non-dietary factors associated with dietary habits, which could confound associations between diet and COPD (see below).

\section{Dietary patterns and confounders}

Most studies obviously try to account for the greatest possible number of confounders when assessing the role of a given nutrient or group of nutrients. Multivariate approaches represent the most widely used statistical tools in this respect. Another interesting and probably complementary way of addressing this issue is the use of clustering techniques, which may reveal that, despite being individually "independently" related to a given medical condition in multivariate analysis, some dietary habits belong to common patterns. This topic has been reviewed recently by DEvLIN et al. [28], who highlighted important methodological issues and reviewed dietary patterns identified in healthy population and several diseases. Altogether, two distinct patterns emerged almost constantly, i.e. the "healthy pattern" (consumption of fruit and vegetables) and the "unhealthy", "Western" or "traditional" pattern (see later). These data underline that the "independence" of factors associated with a given condition should be taken as a relative notion, which may be highly influenced by assessment methods. Conversely, dietary patterns identified by clustering techniques are rather rough in that they may not capture very specific profiles and thus do not allow mechanisms of action to be explored.

Interestingly, the link between cereal fibres and COPD occurrence mentioned previously might be a confounding factor when assessing the relationships between the risk of COPD and bean consumption in Hispanics, since these populations often eat beans with cereals.

\section{Possible confounding by non-dietary factors}

A challenging issue relates to the possible association between a high consumption of the "protective" nutrients listed above and other lifestyle characteristics, including increased physical activity. Indeed, in general, the current evolution of the occidental way of life is criticised for two aspects: a more sedentary lifestyle and dietary deviation towards the Western diet that is rich in meats, refined grains, desserts and French fries [6]. Both promote systemic inflammation and oxidative stress [29]. Interestingly, both also favour the occurrence of the metabolic syndrome, a combination of cardiovascular risk factors that are well known to be associated with COPD [30].

\section{Physical activity in COPD}

Even mild-to-moderate COPD is associated with a decrease in physical activity [31], which is thought to occur primarily as a consequence of dyspnoea induced by increased ventilatory constraints. Indeed, dynamic hyperinflation has been demonstrated in symptomatic patients with mild airflow limitation [32]. 
Exercise intolerance also relates to skeletal muscle dysfunction, characterised by both quantitative (loss of muscle mass) and qualitative (reduced enzymatic capacities) features resulting from sedentariness and probably from systemic consequences of COPD, including inflammation and oxidative stress [33]. Low levels of activity are related to poorer survival in COPD patients, as well as in the general population [33]. Some COPD-specific outcomes (e.g. exacerbations and hospitalisations) are also impaired in patients with low levels of physical activity.

Understanding relationships between diet and physical activity requires two issues to be considered: one is the methods of measurement, the other is the statistical methodology.

\section{Field measurement of physical activity in epidemiology}

The gold standard for physical activity estimation is based on measurements of total energy expenditure by direct calorimetry using doubly labelled water [34]. Physical activity can then be calculated based on total energy expenditure and basal energy expenditure, with the ratio or difference between these variables representing physical activity-related energy expenditure. Such techniques are obviously inappropriate for epidemiological studies, but are to be used to validate field methods. Similarly, behavioural observation (by direct observation or filming) cannot be easily implemented on a large scale, and is actually not fully validated yet as a gold standard. Heart rate and ventilation monitors are influenced by factors other than physical activity [34]. Indirect calorimetry methods, based on the determination of overall oxygen consumption by respirometers, also provide data of debated validity. Therefore, until recently, physical activity assessment for epidemiological purposes mostly relied on questionnaires [35]. Although activity monitors might provide more accurate and reliable (i.e. objective) evaluations, it has long proven difficult to implement their general use in field studies due to cost and feasibility issues, as well as uncertainties regarding the best monitor to choose (pedometer and/or accelerometer). In addition, the need for a conceptual framework in the field of activity measurement has been outlined [36]. In recent years, several epidemiological studies using activity monitors have been published and several initiatives are currently ongoing regarding their use in the field of COPD [37].

\section{Physical activity and respiratory health in the population}

Since physical activity is influenced by lung function levels, cross-sectional studies are not useful in assessing whether the lack of physical activity per se influences the risk of accelerated lung function decline and COPD occurrence. Unfortunately, very few longitudinal studies focused on this question. GARCIA-AYMERICH et al. [38] analysed data from the Copenhagen City Heart Study and found that moderate-to-high physical activity decreased the rate of FEV1 and forced vital capacity decline in smokers, and protected against COPD occurrence. This might relate to the anti-inflammatory properties of physical activity, and some longitudinal studies indeed showed that baseline C-reactive protein levels, which rise in response to inflammation, are related to subsequent lung function decline [39]. However, this relationship was found to persist independently of physical activity levels, showing that systemic inflammation cannot be considered as a proxy for physical activity. In addition, other results challenge these findings [40].

\section{Clustering of physical activity and diet}

Activity assessment tools allow refinement of diet-physical activity clustering assessment. Corresponding data were summarised in a systemic review published in 2011, which included 56 studies, most dealing with children and adolescents (19 studies in children, 26 in adolescents and 11 in adults). Most studies used cross-sectional designs, and confirm the relationship between sedentariness (and, in parallel, screen time) and unhealthy dietary patterns, as illustrated by higher consumption of energy-dense snacks and less consumption of fruit and vegetables, consistent across age groups [41]. The strength of the relationship between diet and activity was small-to-moderate overall, and slightly less in adults than in younger age groups. Most studies of the relationships between diet and lung function/COPD adjust analyses for physical activity [17] but, again, residual confounding cannot be excluded, especially when measures are only questionnaire based.

\section{Another component of the "unhealthy behaviour cluster": adiposity}

Nutritional status is obviously linked to both dietary habits and physical activity. The metabolic syndrome and, more specifically, abdominal adiposity has been associated with lung function impairment [42]. Data regarding the relationship between body mass index and lung function decline are conflicting. These findings are interesting since abdominal adiposity is more specifically associated with systemic inflammation than global obesity. Altogether, it is tempting to hypothesise that a diet poor in antioxidants, low level of physical activity and abdominal adiposity are inter-related, and concur in accelerating lung function decline through inflammation-mediated mechanisms. 


\section{Conclusions}

In summary, as a whole, no nutrient fully satisfies the criteria for causality defined by HILL [2]. Existing data indicate that an increase in the consumption of vegetables, fruit and (although with less evidence) fibre may contribute to the prevention of COPD. However, because of the paucity of longitudinal data, these relationships can only be considered possible. Inversely, based on some data that need to be confirmed, cured and red meats, desserts, and refined grains constitute potential risk factors for COPD. There is no clear evidence for beans, which has been specifically considered in only one study. Additionally, in Latin American countries, beans are often eaten with other fibres (specifically cereal fibres) and without cured meat, which could protect against COPD. Such habits are also very common in less affluent classes where a sedentary lifestyle has, so far, been exceptional.

Only results from prospective cohort studies, together with those from intervention studies on the mechanisms of action of beans and related diet, will provide a sound basis for future evidence-based evaluations of the preventive potential of bean consumption regarding various chronic diseases including COPD. These studies have to take into account potential confounders such as smoking, physical activity, nutritional status and other at-risk behaviours.

\section{References}

Young RP, Hopkins RJ. A review of the Hispanic paradox: time to spill the beans? Eur Respir Rev 2014; 23: 439-449. Hill AB. The environment and disease: association or causation? Proc R Soc Med 1965; 58: 295-300.

Glass TA, Goodman SN, Hernán MA, et al. Causal inference in public health. Annu Rev Public Health 2013; 34: 61-75. Rothman KJ, Greenland S. Causation and causal inference in epidemiology. Am J Public Health 2005; 95: Suppl. 1, S144-S150

Potischman N, Weed DL. Causal criteria in nutritional epidemiology. Am J Clin Nutr 1999; 69: 1309S-1314S. Fonseca Wald EL, van den Borst B, Gosker HR, et al. Dietary fibre and fatty acids in chronic obstructive pulmonary disease risk and progression: a systematic review. Respirology 2014; 19: 176-184

7 Hanson C, Rutten EPA, Wouters EF, et al. Diet and vitamin D as risk factors for lung impairment and COPD. Transl Res 2013; 162: 219-236.

Barnes PJ. New therapies for asthma: is there any progress? Trends Pharmacol Sci 2010; 31: 335-343.

9 Yarnell JW, Fehily AM, Milbank JE, et al. A short dietary questionnaire for use in an epidemiological survey: comparison with weighed dietary records. Hum Nutr Appl Nutr 1983; 37: 103-112.

10 Hirayama F, Lee AH, Binns CW. Dietary factors for chronic obstructive pulmonary disease: epidemiological evidence. Expert Rev Respir Med 2008; 2: 645-653.

11 Butland BK, Fehily AM, Elwood PC. Diet, lung function, and lung function decline in a cohort of 2512 middle aged men. Thorax 2000; 55: 102-108.

12 Tabak C, Smit HA, Heederik D, et al. Diet and chronic obstructive pulmonary disease: independent beneficial effects of fruits, whole grains, and alcohol (the MORGEN study). Clin Exp Allergy 2001; 31: 747-755.

13 Kelly Y, Sacker A, Marmot M. Nutrition and respiratory health in adults: findings from the health survey for Scotland. Eur Respir J 2003; 21: 664-671.

14 Celik F, Topcu F. Nutritional risk factors for the development of chronic obstructive pulmonary disease (COPD) in male smokers. Clin Nutr 2006; 25: 955-961.

15 Miedema I, Feskens EJ, Heederik D, et al. Dietary determinants of long-term incidence of chronic nonspecific lung diseases. The Zutphen Study. Am J Epidemiol 1993; 138: 37-45.

16 Walda IC, Tabak C, Smit HA, et al. Diet and 20-year chronic obstructive pulmonary disease mortality in middleaged men from three European countries. Eur J Clin Nutr 2002; 56: 638-643.

17 Varraso R, Willett WC, Camargo CA Jr. Prospective study of dietary fiber and risk of chronic obstructive pulmonary disease among US women and men. Am J Epidemiol 2010; 171: 776-784.

18 Varraso R, Fung TT, Hu FB, et al. Prospective study of dietary patterns and chronic obstructive pulmonary disease among US men. Thorax 2007; 62: 786-791.

19 Hirayama F, Lee AH, Binns CW, et al. Do vegetables and fruits reduce the risk of chronic obstructive pulmonary disease? A case-control study in Japan. Prev Med 2009; 49: 184-189.

20 Watson L, Margetts B, Howarth P, et al. The association between diet and chronic obstructive pulmonary disease in subjects selected from general practice. Eur Respir J 2002; 20: 313-318.

21 McKeever TM, Lewis SA, Cassano PA, et al. Patterns of dietary intake and relation to respiratory disease, forced expiratory volume in $1 \mathrm{~s}$, and decline in 5-y forced expiratory volume. Am J Clin Nutr 2010; 92: 408-415.

22 Varraso R, Jiang R, Barr RG, et al. Prospective study of cured meats consumption and risk of chronic obstructive pulmonary disease in men. Am J Epidemiol 2007; 166: 1438-1445.

23 De Batlle J, Mendez M, Romieu I, et al. Cured meat consumption increases risk of readmission in COPD patients. Eur Respir J 2012; 40: 555-560.

24 Varraso R, Fung TT, Barr RG, et al. Prospective study of dietary patterns and chronic obstructive pulmonary disease among US women. Am J Clin Nutr 2007; 86: 488-495.

25 Hirayama F, Lee AH, Binns CW, et al. Soy consumption and risk of COPD and respiratory symptoms: a casecontrol study in Japan. Respir Res 2009; 10: 56.

26 Tsiligianni IG, van der Molen T. A systematic review of the role of vitamin insufficiencies and supplementation in COPD. Respir Res 2010; 11: 171.

27 Broekhuizen R, Wouters EF, Creutzberg EC, et al. Polyunsaturated fatty acids improve exercise capacity in chronic obstructive pulmonary disease. Thorax 2005; 60: 376-382.

28 Devlin UM, McNulty BA, Nugent AP, et al. The use of cluster analysis to derive dietary patterns: methodological considerations, reproducibility, validity and the effect of energy mis-reporting. Proc Nutr Soc 2012; 71: 599-609. 
Hopkinson NS, Polkey MI. Does physical inactivity cause chronic obstructive pulmonary disease? Clin Sci (Lond) 2010; 118: 565-572.

30 Fabbri LM, Rabe KF. From COPD to chronic systemic inflammatory syndrome? Lancet 2007; 370: 797-799.

31 Pitta F, Troosters T, Spruit MA, et al. Characteristics of physical activities in daily life in chronic obstructive pulmonary disease. Am J Respir Crit Care Med 2005; 171: 972-977.

32 Ofir D, Laveneziana P, Webb KA, et al. Mechanisms of dyspnea during cycle exercise in symptomatic patients with GOLD stage I chronic obstructive pulmonary disease. Am J Respir Crit Care Med 2008; 177: 622-629.

33 Troosters T, van der Molen T, Polkey M, et al. Improving physical activity in COPD: towards a new paradigm. Respir Res 2013; 14: 115.

34 Westerterp KR. Assessment of physical activity: a critical appraisal. Eur J Appl Physiol 2009; 105: 823-828.

35 Shephard RJ, Aoyagi Y. Measurement of human energy expenditure, with particular reference to field studies: an historical perspective. Eur J Appl Physiol 2012; 112: 2785-2815.

36 Gimeno-Santos E, Frei A, Dobbels F, et al. Validity of instruments to measure physical activity may be questionable due to a lack of conceptual frameworks: a systematic review. Health Qual Life Outcomes 2011; 9: 86.

37 Rabinovich RA, Louvaris Z, Raste Y, et al. Validity of physical activity monitors during daily life in patients with COPD. Eur Respir J 2013; 42: 1205-1215.

38 Garcia-Aymerich J, Lange P, Benet M, et al. Regular physical activity modifies smoking-related lung function decline and reduces risk of chronic obstructive pulmonary disease: a population-based cohort study. Am J Respir Crit Care Med 2007; 175: 458-463.

39 Ahmadi-Abhari S, Kaptoge S, Luben RN, et al. Longitudinal association of C-reactive protein and lung function over 13 years: The EPIC-Norfolk study. Am J Epidemiol 2014; 179: 48-56.

40 Fogarty AW, Jones S, Britton JR, et al. Systemic inflammation and decline in lung function in a general population: a prospective study. Thorax 2007; 62: 515-520.

41 Pearson N, Biddle SJH. Sedentary behavior and dietary intake in children, adolescents, and adults. A systematic review. Am J Prev Med 2011; 41: 178-188.

42 Leone N, Courbon D, Thomas F, et al. Lung function impairment and metabolic syndrome: the critical role of abdominal obesity. Am J Respir Crit Care Med 2009; 179: 509-516. 